Sebastian M. Arnold

Ralph Strecker

Klaus Scheffler

Joachim Spreer

Jörg Schipper

Hartmut P. H. Neumann

Joachim Klisch

\section{Dynamic contrast enhancement of paragangliomas of the head and neck: evaluation with time-resolved 2D MR projection angiography}

Received: 19 March 2002

Revised: 5 August 2002

Accepted: 5 September 2002

Published online: 1 November 2002

(C) Springer-Verlag 2002

S.M. Arnold ( $)$ J. Spreer · J. Klisch

Department of Neuroradiology,

University of Freiburg,

79106 Freiburg, Germany

e-mail: sarnold@uhbs.ch

Tel.: +41-61-2652525

Fax: +41-61-2655471

R. Strecker $\cdot$ K. Scheffler

Department of MR Physics,

University of Freiburg,

79106 Freiburg, Germany

S.M. Arnold

Department of Radiology,

University Hospital, Petersgraben 4,

4031 Basel, Switzerland

\section{J. Schipper}

Department of Otolaryngology,

University of Freiburg,

79106 Freiburg, Germany

H.P.H. Neumann

Department of Nephrology and Hypertension, University of Freiburg,

79106 Freiburg, Germany

\begin{abstract}
Our objective was to evaluate tumor hemodynamics of paragangliomas of the head and neck. A 2D magnetic resonance projection angiography (MRPA) protocol was developed. Six patients with histologically proven paragangliomas were included in the study. The sequence protocol and data post-processing procedure were optimized in view of a high temporal resolution and maximum contrast-to-noise ratio. Image-time series of two freely orientated slabs could be obtained. Correlation analysis was performed to generate selective arterial and venous weighted angiographic images. Glomus tumors showed a rapid and intense homogeneous enhancement following the intravenous administration of contrast material. The smallest tumors investigated measured $10 \mathrm{~mm}$ and could be visualized clearly. Time-resolved analysis of the tumor enhancement revealed an early bolus arrival within the vascular bed of the tumor and prolonged
\end{abstract}

incomplete washout indicating blood pooling. The dynamic contrast enhancement of glomus tumors can be evaluated non-invasively with MRPA providing a high temporal resolution and high image quality. The characteristic contrast enhancement of glomus tumors can be helpful in the diagnostic workup of lesions that may mimic glomus tumors.

Keywords Paraganglioma - Glomus tumor $\cdot$ MR imaging $\cdot$ MR projection angiography

\section{Introduction}

Paragangliomas are rare highly vascularized tumors in adults. The most common locations of head and neck are the carotid space and the jugular fossa. Familial paragangliomas appear in $7 \%$ of cases [1]. Recently, a gene focus in families with inherited paragangliomas or glomus tumors has been identified [2, 3]. Despite paragangliomas are classified as benign tumors WHO grade I, dis- tant metastases have been published $[4,5]$. On the other hand, $10 \%$ of glomus tumors may be multicentric in origin. Neuroimaging shows a well-circumscribed, strongly enhancing mass associated with paraganglionic structures indicating abundant blood supply. Both CT and MR may show erosions of adjacent bony structures. Paragangliomas are of variable size, encapsulated, with non-invasive but expansive growth. A "salt-and-pepper" appearance of the tumor due to flow voids and small 
areas of subacute hemorrhage is characteristic [6, 7]. Time-resolved contrast-enhanced magnetic resonance angiography is increasingly gaining interest since it offers anatomical and functional information about the vascular system $[8,9,10]$.

In this study we evaluated magnetic resonance projection angiography (MRPA) for the visualization of glomus tumors of the jugular foramen and the carotid bifurcation. We propose that MRPA is a valuable method for detection of glomus tumors.

\section{Materials and methods}

Six patients suffering from histologically proven paragangliomas 4 glomus caroticum tumors ( 3 unilateral, 1 bilateral) and 2 glomus jugulare tumors - were investigated in the study. The tumor size varied between 1 and $5 \mathrm{~cm}$. All patients gave informed consent to the study protocol. The examinations were performed on a 1.5-T scanner (Magnetom Vision, Siemens, Erlangen, Germany) using the standard whole-body gradient set with $25 \mathrm{mT} / \mathrm{m}$ amplitude and $600 \mu$ s rise time. Images were acquired using the standard cp head coil or cp neck array. A radio-frequency-spoiled snapshot fast lowangle shot (FLASH) sequence with a slice selective ShinnarLe Roux excitation pulse and a minimum TE of $1.5 \mathrm{~ms}$ and TR of $4.2 \mathrm{~ms}$ was used. The read out acquisition time was $1.56 \mathrm{~ms}$ for 160 sampling points, starting 32 points before the echo center. A minimum field of view of $220 \mathrm{~mm}$ was used. Slice thickness and orientation was selected freely including the whole volume of interest, i.e., both hemispheres. The typical slice thickness was $80 \mathrm{~mm}$. A significant improvement of the temporal resolution could be achieved by the use of view sharing and temporal interpolation [11]. Using a matrix size of $256 \times 256$ a temporal resolution of four images per second was possible. In a double-slab protocol two images per second could be acquired, respectively. A high-resolution protocol permitted a matrix size of $512 \times 512$. The images were inline mask subtracted to a temporal averaged image acquired prior to contrast bolus arrival. This technique was used to provide a better delineation of small vessels and to suppress signal cancellations caused by magnitude subtraction at boundary areas of different susceptibilities. Correlation analysis was used to selectively derive functional angiograms of the arterial and venous phase with a significantly improved signal-to-noise ratio (SNR) [12]. Contrast agent administration of 10-15 ml gadoteridol (Prohance, Byk Gulden, Germany) was started with a 10-s delay using a power injector with a flow of 4-5 ml/s flushed by $20 \mathrm{ml}$ of saline. The total acquisition time was below $1 \mathrm{~min}$.

\section{Results}

After contrast application, in all cases an intense and homogeneous tumor blush was demonstrated (Fig. 1). In the case of larger tumors the carotid bifurcation was splayed. Tumor borders were well defined; however, in extended lesions the carotid bifurcation could be obscured by the tumor. In these cases conventional T1- and T2-weighted images were superior in demonstrating the course of the adjacent vessels. In small tumors biplane illustrations of the carotid bifurcation in coronal and sagittal orientation were advantageous. Concerning contrast dynamics, all glomus tumors showed an early and rapid

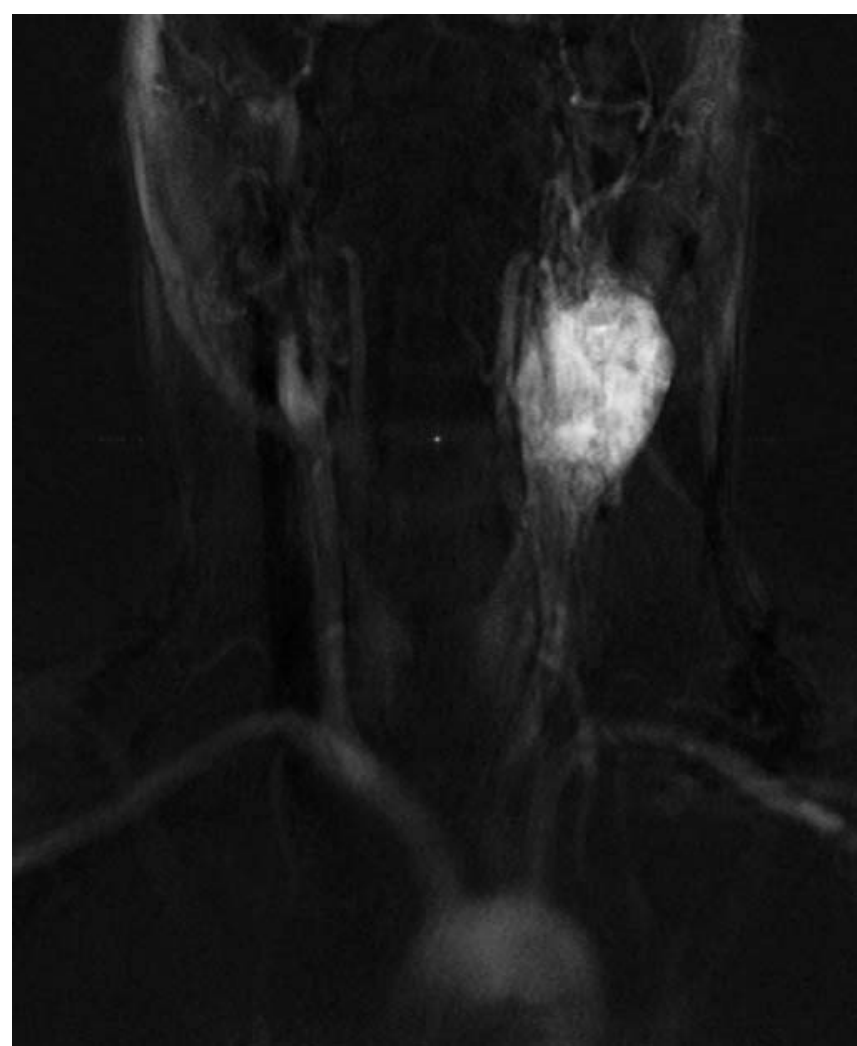

Fig. 1 Coronal magnetic resonance projection angiography image of a large left-sided glomus caroticum tumor in early arterial phase (matrix size $256 \times 256$, data acquisition of four images per second)

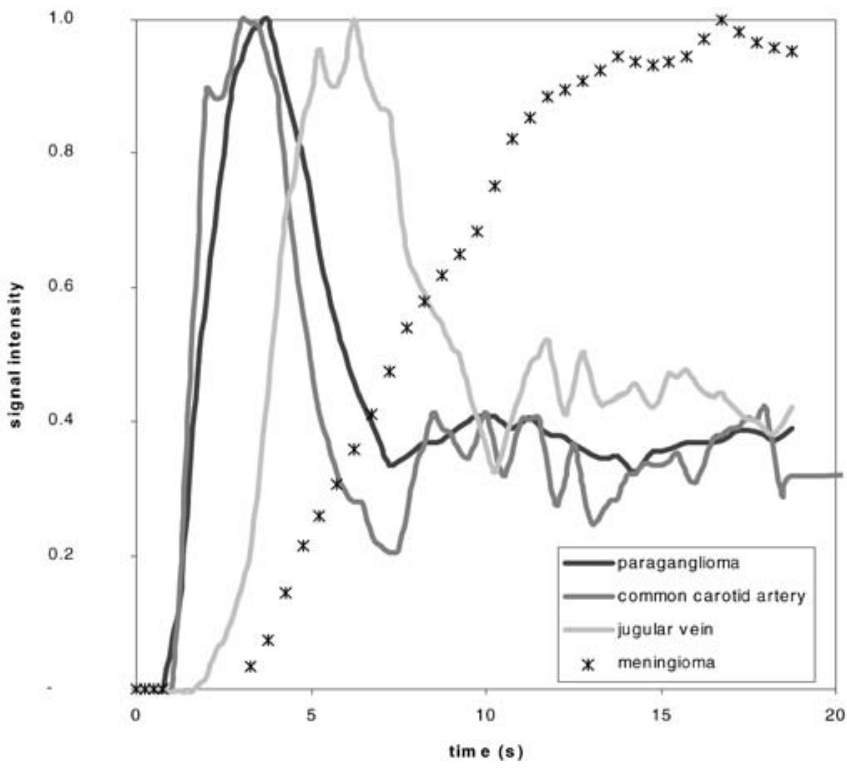

Fig. 2 Normalized signal intensity/time curves by setting the maximum signal intensity to unity are plotted. Signal/time curves are shown for three different regions of interest placed in the common carotid artery, the left-sided paraganglioma and the jugular vein, respectively. For comparison, time-dependent enhancement of an intracranial meningioma of another patient is outlined (asterisks) 
enhancement after contrast administration very similar to the arterial vascular enhancement (Fig. 2); however, due to summation effects, the maximum of intensity could differ significantly. In the venous phase the signal intensity of glomus tumors persisted on a higher level indicating blood pooling. Postprocessing enables a selective visualization of the tumor with complete suppression of venous signal. In one patient we performed a follow-up examination after combined preoperative tumor embolization and surgery, which found the tumor had completely vanished. Contrast enhancement of the glomus jugulare tumors was quite similar to the early enhancement in glomus caroticum tumors; however, as the growth pattern of glomus tumors in the region of the jugular foramen is known to be highly variable with possibly extraand intracranial compartments, the whole extent of the tumor is more ill defined. Furthermore, both tumors were investigated after tumor surgery with clinical evidence of tumor recurrence. For this reason additional scar formation could not be excluded.

\section{Discussion}

The diagnostic value of functional imaging in the assessment of masses of the head and neck suggestive of paragangliomas is still under debate. In our study we could show a typical time-dependent enhancement pattern of glomus caroticum tumors and glomus jugulare tumors using MRPA. The selective imaging of vascular and tumor enhancement easily points out also small lesions, which might open a new diagnostic field in screening investigations. The MRPA is a non-invasive technique with minimal time demand and could be simply included within a routine clinical MRI scanning protocol.

Dynamic MR digital subtraction angiography by using fast acquisition, contrast enhancement, and complex subtraction was first proposed by Wang et al. [10]. The sequence protocol we used in our study was published by Hennig et al. [9] and has already reached a temporal resolution in the subsecond domain. An improved SNR of the projection images up to a factor three could be achieved by using correlation analysis similar to the algorithms used in functional MRI [12]. Some applications of the method in fast MR angiography with subsecond resolution have been published recently $[13,14,15,16]$.

The combination of MR projection angiography with view sharing and inline background subtraction offers several advantages [11]. Firstly, the SNR in the single images of the time series is improved due to temporal interpolation without reducing spatial resolution. Secondly, steady-state magnetization is increased and leads to a further gain in the SNR. Thirdly, the matrix size can be increased to $512 \times 512$ while retaining the temporal resolution in the subsecond domain. The increase of temporal resolution due to view sharing allows multiple slab measurement with subsecond temporal resolution within one examination.

In a study by Vogl et al. [17] contrast enhancement of glomus tumors was assessed dynamically by the use of a single-slice gradient-recalled sequence with a temporal resolution of $6 \mathrm{~s}$. They found a dropout effect in form of an initial dip during early enhancement, which they concluded might be a specific sign for glomus tumors. They postulated that the initial signal decline might be the result of susceptibility effects of the highly concentrated intravascular contrast agent. Our data could not confirm such a strong susceptibility effect after intravenous contrast agent administration, although it is a well-known effect after intra-arterial contrast administration in MR angiography [18]. Naegele et al. [19] used a fast snapshot-FLASH single-slice imaging technique allowing dynamic contrast enhancement imaging with measurement times of $1 \mathrm{~s}$ per image. They could demonstrate a very early contrast enhancement of glomus tumors as compared to neurinomas and meningiomas. Our study confirms that glomus caroticum tumors and glomus jugulare tumors show a typical temporal contrast enhancement (Fig. 2). The high vascularization of the tumors with high blood flow and sometimes arterial-venous shunting leads to nearly simultaneous contrast approach in tumor tissue and supplying arteries. Due to the summation effect within the slice, the absolute signal of tumor and adjacent arteries could vary considerably. Concerning the whole extent of the tumor blush, however, MRPA was postulated to be superior to conventional angiography [15]. Particularly tumors fed by several arterial systems may only become partially opacified in conventional angiography. Non-enhanced blood from other feeding vessels may lead to a selective washout effect of the injected contrast agent. Due to non-selective bolus application, MRPA visualizes all vessels within the selected volume.

In comparison with the arterial concentration time curve, the venous signal of glomus tumors was prolonged on a higher level. One explanation is the delayed washout of contrast agent from the tumor into the vascular bed. On the other hand, the recirculation of contrast agent may explain an increase in baseline signal indicated by the signal approach of the mean arterial and venous signal. To some unknown extent, disruption of blood-brain barrier should be taken into account.

In the case of small tumor size, there could be a considerable overlap with the bulb of the internal carotid artery, which can obscure the tumor in the carotid bifurcation. The use of an additional second plane can overcome this limitation by taking into account the anatomical configuration of the carotid bifurcation. Although MRPA is not able to offer spatial depth information, biplane imaging may help to evaluate, for example, the carotid bifurcation in detail, and to distinguish it from other carotid-space pathologies. 
Scar formation after surgical approach is often difficult to differentiate from recurrent tumor or residual tumor [20, 21]. Grees et al. [22] used a T1 fast-field-echo sequence to study dynamic contrast enhancement after surgery for glomus jugulare tumors. They found clearly different dynamic contrast appearance of scars and re- current or rest tumor. Whereas recurrent or rest tumor showed a strong enhancement and a washout phenomenon, scars showed much less contrast enhancement persisting on a low level. Although we could not demonstrate scar formation in our patients, MRPA gave additional information in these difficult cases.

\section{References}

1. Lantos PL, Vandenberg SR, Keihues P (1997) Tumours of the nervous system. In: Graham DI, Lantos PL (eds) Greenfield's neuropathology, 6th edn. Arnold, London, pp 583-795

2. Baysal BE, Ferrell RE, Willett-Brozick JE et al. (2000) Mutations in SDHD, a mitochondrial complex II gene, in hereditary paraganglioma. Science 287:848-851

3. Niemann S, Müller U (2000) Mutations in SDHC cause autosomal dominant paraganglioma, type 3 . Nat Genet 26:268-270

4. Brewis C, Bottrill ID, Wharton SB et al. (2000) Glomus jugulare tumour with metastases to cervical lymph nodes. J Laryngol Otol 114:67-69

5. Mossner R, Keidel M (2000) Malignant pheochromocytoma with progressive paraparesis in von Hippel-Lindau disease. Eur J Neurol 7:439-442

6. Osborn AG, Tong KA (1996) Handbook of neuroradiology: brain and skull, 2nd edn. Mosby, St. Louis

7. Rao AB, Koeller KK, Adair CF (1999) Paragangliomas of the head and neck: radiologic-pathologic correlation. Radiographics 19:1605-1632

8. Korosec FR, Frayne R, Grist TM et al. (1996) Time-resolved contrast-enhanced 3D MR angiography. Magn Reson Med 36:345-351
9. Hennig J, Scheffler K, Laubenberger J et al. (1997) Time-resolved projection angiography after bolus injection of contrast agent. Magn Reson Med 37:341-345

10. Wang Y, Johnston DL, Breen JF et al. (1996) Dynamic MR digital subtraction angiography using contrast enhancement, fast data acquisition, and complex subtraction. Magn Reson Med 36:551-556

11. Strecker R, Arnold S, Scheffler K et al. (2001) In: Proc Joint Annual Meeting, ISMRM and ESMRMB, Glasgow, p 301

12. Strecker R, Scheffler K, Klisch J et al. (2000) Fast functional MRA using time-resolved projection MR angiography with correlation analysis. Magn Reson Med 43:303-309

13. Klisch J, Strecker R, Hennig J et al. (2000) Time-resolved projection MRA: clinical application in intracranial vascular malformations. Neuroradiology 42:104-107

14. Wetzel SG, Bilecen D, Lyrer P et al. (2000) Cerebral dural arteriovenous fistulas: detection by dynamic MRPA. Am J Roentgenol 174:1293-1295

15. Yoshikawa T, Aoki S, Hori M et al. (2000) Time-resolved two-dimensional thick-slice magnetic resonance digital subtraction angiography in assessing brain tumors. Eur Radiol 10:736-744

16. Griffiths PD, Hoggard N, Warren DJ et al. (2000) Brain arteriovenous malformations: assessment with dynamic MR digital subtraction angiography. Am J Neuroradiol 21:1892-1899
17. Vogl TJ, Mack MG, Juergens M et al. (1993) Skull base tumors: gadodiamide injection-enhanced MR imaging: dropout effect in the early enhancement pattern of paragangliomas versus different tumors. Radiology 188:339-348

18. Serfaty JM, Atalar E, Declerck J et al. (2000) Real-time projection MR angiography: feasibility study. Radiology 217:290-295

19. Naegele T, Petersen D, Klose U et al. (1993) Dynamic contrast enhancement of intracranial tumors with snapshotFLASH MRI. Am J Neuroradiol 14:89-98

20. Schumacher A, Jonas M, Rummeny E et al. (1996) Diagnosis of recurrence of a glomus carotid artery tumor by somatostatin receptor scintigraphy. Nuklearmedizin 35:38-41

21. Konig H, Kurtz B, Strohm M (1984) High-resolution and dynamic computed tomography in the diagnosis of glomus tympanicum and glomus jugulare tumors. Fortschr Geb Roentgenstr Nuklearmed 141:642-646

22. Grees H, Bentzien S, Tomandl B et al. (2000) Rezidivdiagnostik von Glomusjugulare-Tumoren mittels dynamischer Kontrastmittel-MR-Anflutung. Fortschr Geb Roentgenstr Nuklearmed 172:91 